\title{
Patients with High-Grade Gliomas and Café-au-Lait Macules: Is Neurofibromatosis Type 1 the Only Diagnosis?
}

W e read with great interest the publication "High-Grade Gliomas in Children with Neurofibromatosis Type 1: Literature Review and Illustrative Cases" by Spyris et al. ${ }^{1}$ As discussed by the authors, high-grade gliomas in children may be associated with neurofibromatosis type 1 (NF-1). However, several other cancer predisposition syndromes, including constitutional mismatch repair deficiency (CMMRD), also increase the risk of childhood high-grade glioma. Most important, not only does the spectrum of CMMRDassociated malignancies overlap that reported for NF-1 but patients with CMMRD frequently also show nonmalignant features of NF-1. ${ }^{2}$ This phenotypic overlap between NF-1 and CMMRD challenges the clinical diagnosis of NF-1 in a patient with pediatric highgrade glioma and, overall, our current knowledge on associations between NF-1 and rare childhood malignancies. ${ }^{3}$

Because patients with CMMRD-associated high-grade gliomas may benefit from immunotherapy with anti-programmed cell death protein 1 drugs, ${ }^{4}$ it is key to explore extensively the underlying genetic disease by molecular genetic diagnosis. Distinction between CMMRD and NF-1 in this setting also has implications for further cancer surveillance of the patient, recurrence risk in siblings, and Lynch syndrome-associated cancer risks in the extended family.

In this respect, the cases presented by Spyris et $\mathrm{al}^{1}$ are illustrative of the diagnostic challenge that may occur in this setting. The article reports 5 patients with a brain tumor who were "diagnosed with NF-1 in accordance with the diagnostic criteria." Considering that the message of the article is to raise awareness of the occurrence of high-grade glioma in children with NF-1, we would be very interested in a description of the phenotype and family history of these children and what NF-1 criteria were used to state the clinical diagnosis of NF-1. It would also be of interest to know whether focal abnormal signal intensities (FASIs), a very common finding in children with NF-1, were present. None of the reported patients had visible FASIs on the MRIs presented in the figures. Three patients (patients 1, 2, and 4) had malignant tumors molecularly consistent with diffuse midline $H 3 K 27 M$-mutated gliomas, which have not been described in children with NF-1. 5,6 "Skin spots," presumably café-au-lait macules, were explicitly re-

http://dx.doi.org/10.3174/ajnr.A6058 ported only for patient 4 . Even the tumors most typically associated with NF-1 (ie, optic pathway glioma and pilocytic astrocytoma) as diagnosed in patients 3 and 5 have been described in CMMRD. ${ }^{3}$ Taken together, the diagnosis of NF-1 is questionable in at least some of the patients. Considering the impact of the diagnosis of the underlying genetic disease on the treatment of affected patients, it would be of major importance to genetically confirm the diagnosis of NF-1 in these 5 patients.

To conclude, we strongly recommend that in all children with high-grade gliomas and a phenotype reminiscent of NF-1, this diagnosis should be confirmed as the underlying disease by identification of a clearly pathogenic germline NF1 mutation. In the absence of this confirmation, CMMRD should be excluded by mutation analysis of the $M M R$ genes and/or by immunohistochemistry showing the expression of all $4 \mathrm{MMR}$ proteins in the tumor or by appropriate molecular and functional assays. ${ }^{3}$ A correct diagnosis in these children will be important to adapt the therapeutic strategy, surveillance, and genetic counseling as stated in recent consensus papers. A definite genetic diagnosis of NF-1 and exclusion of CMMRD in future patients are necessary to substantiate the reported association of NF-1 with childhood highgrade gliomas because this is currently largely based on patients diagnosed only on clinical criteria (for review see Wimmer et $\mathrm{al}^{3}$ ) and, therefore, challenged by our current knowledge of the phenotypic overlap between CMMRD and NF-1.

Disclosures: Eric Legius_UNRELATED: Grants/Grants Pending: University of Leuven, Comments: I applied for a new grant at the University of Leuven for studying behavior in a genetic mouse model. I received a grant from the University of Leuven for studying autistic behavior in a mouse model.* *Money paid to the institution.

\section{REFERENCES}

1. Spyris CD, Castellino RC, Schniederjan MJ, et al. High-grade gliomas in children with neurofibromatosis type 1: literature review and illustrative cases. AJNR Am J Neuroradiol 2019;40:366-69 CrossRef Medline

2. Wimmer K, Kratz CP, Vasen HF, et al; EU-Consortium Care for CMMRD (C4CMMRD). Diagnostic criteria for constitutional mismatch repair deficiency syndrome: suggestions of the European consortium "care for CMMRD" (C4CMMRD). J Med Genet 2014;51: 355-65 CrossRef Medline

3. Wimmer K, Rosenbaum T, Messiaen L. Connections between constitutional mismatch repair deficiency syndrome and neurofibromatosis type 1. Clin Genet 2017;91:507-19 CrossRef Medline 
4. Bouffet E, Tabori U, Bartels U. Paediatric ependymomas: should we avoid radiotherapy? Lancet Oncol 2007;8:665-66 CrossRef Medline

5. Mackay A, Burford A, Carvalho D, et al. Integrated molecular metaanalysis of 1,000 pediatric high-grade and diffuse intrinsic pontine glioma. Cancer Cell 2017;32:520-37.e5 CrossRef Medline

6. D'Angelo F, Ceccarelli M, Tala, et al. The molecular landscape of glioma in patients with neurofibromatosis 1. Nat Med 2019;25:176-87 CrossRef Medline

(D). Guerrini-Rousseau Department of Pediatric and Adolescents Oncology Gustave Roussy Cancer Center Paris-Saclay University Villejuif, France (1) M. Suerink Department of Clinical Genetics Leiden University Medical Centre Leiden, the Netherlands
(D). Grill

Department of Pediatric and Adolescents Oncology Gustave Roussy Cancer Center Paris-Saclay University Villejuif, France (1)E. Legius Department of Human Genetics University Hospital Leuven and KU Leuven Leuven, Belgium (1) K. Wimmer

Division of Human Genetics Medical University Innsbruck Innsbruck, Austria (1) L. Brugières

Department of Pediatric and Adolescents Oncology Gustave Roussy Cancer Center Paris-Saclay University Villejuif, France 